\title{
ThASCEENDER
} Trascender, Contabilidad y Gestión Núm. 12 (septiembre - diciembre del 2019). Universidad de Sonora. Departamento de Contabilidad. ISSN: 2448-6388. Reserva de Derechos 04-2015-04172070800-203.

\section{La profesionalización del Poder Legislativo en Sonora, ¿calidad o cantidad? Periodo 2009-2018}

The professionalization of the Legislative Power in Sonora, quality or quantity? Period 2009-2018

\author{
Luis Carlos Rodríguez Montaño ${ }^{1}$
}

Recibido: 12 de julio de 2019 .

Aceptado: 19 de agosto de 2019.

DOI: https://doi.org/10.36791/tcg.v11i0.69

JEL: H7. Administración estatal. H70 Generalidades.

\section{Resumen}

El objetivo principal del presente trabajo de investigación es revisar el contexto del sistema de evaluación del desempeño legislativo en Sonora, así como analizar los resultados que se dieron con la implementación de dicho sistema en el periodo 20092018. Existe una crítica persistente a la falta de profesionalización de legisladores y legisladoras en el poder legislativo federal y los congresos locales. Esta situación no es ajena a la percepción que se tiene de los integrantes del congreso sonorense. Dicha crítica se sustenta en cuanto a que diputados y diputadas carecen de preparación en general en relación al trabajo que desempeñan. En principio, divisamos dos partes de un mismo problema; se afirma que el congreso presenta iniciativas de mala calidad, y por otra parte que incumple con los requisitos mínimos que le impone la ley orgánica. Derivado de dicha afirmación, nos abocaremos a analizar la metodología del sistema de evaluación, ofreciendo la descripción y análisis de los resultados.
Palabras clave: Poder Legislativo, indicadores, evaluación.

\begin{abstract}
The main objective of this research work is to review the context of the legislative performance evaluation system in Sonora, as well as to analyze the results that occurred with the implementation of said system in the period 2009-2018. There is a persistent criticism of the lack of professionalization of legislators in the federal legislature and local congresses. This situation is not unrelated to the perception of the members of the Sonoran congress. This criticism is based on the fact that deputies lack general preparation in relation to the work they perform. In principle, we see two parts of the same problem; it is stated that the congress presents initiatives of poor quality, and on the other hand that it does not comply with the minimum requirements
\end{abstract}

\footnotetext{
${ }^{1}$ Luis Carlos Rodríguez Montaño. Es candidato a Doctor por El Colegio de Sonora en Teoría y Análisis de Asuntos Públicos. Profesor de Asignatura del Departamento de Sociología y Administración Pública de la Universidad de Sonora. Correo: luis.rodriguez@unison.mx
} 
imposed by the organic law. Derived from this statement, we will focus on analyzing the methodology of the evaluation system, offering the description and analysis of the results.

Keywords: legislature, indicator, evaluation.

\section{Introducción}

El trabajo que aquí se presenta tiene sus raíces y se deprende de la cultura de la transparencia y rendición de cuentas, la cual tiene sus orígenes en México en el año 2002 con la aprobación de la ley federal de transparencia y acceso a la información pública, y que, a su vez, en el estado de Sonora en el año 2005 se armonizó con nuestra constitución local lo que derivó en la aprobación de la ley estatal de transparencia. Los congresos locales en general, y el de Sonora en particular, eran entes que por generalidad no eran evaluados ya que su trabajo era de corte cualitativo, de igual forma, los congresos locales hasta antes de la última década del siglo $\mathrm{XX}$ fueron cuerpos colegiados que se percibían más bien opacos en el radar de los ciudadanos, es decir, no estaba muy claro cuáles eran las funciones que se realizaban y tampoco rendían informes que permearan en el conocimiento general de la población.

La evaluación del desempeño en las organizaciones es la manera a través de la cual se le puede dar seguimiento a los objetivos, competencias y la profesionalización de los recursos humanos que pertenecen a una organización. La evaluación del desempeño, en esencia es comparar el trabajo actual con lo anterior de un trabajador en relación con sus propios estándares (Dessler y Varela, 2011, 222). Generalmente las evaluaciones del desempeño se utilizaban en el ámbito de la empresa privada, ya que dichas organizaciones como punto primordial median la productividad de sus empleados, pero este tipo de mediciones no se llevaban a cabo en la esfera del sector público. Los contextos democráticos son tendientes a adoptar una cultura de la evaluación, al respecto Mény y Thoening (1992) aseguran que una evaluación es un juicio hecho sobre un dato con referencia a un valor, derivado de esto se aplica una cuestión entre la alternativa de aplicar y combinar de manera adecuada los elementos cuantitativos y cualitativos de una evaluación.

Esta nueva modalidad de evaluación del desempeño legislativo intenta evaluar la productividad del trabajo de los legisladores sonorenses de acuerdo a las tareas que les competen principalmente, una de las principales es la configuración y la presentación de iniciativas de ley o de decreto, el cual es un trabajo de observancia cualitativa, de tal forma que, la tarea del nuevo sistema de evaluación era darle una especie de calificación al trabajo que la misma ley orgánica le obligaba al congreso. En el texto podremos apreciar que existen indicadores que por su naturaleza se pueden evaluar a priori, como lo son las asistencias a las sesiones del pleno o de las comisiones, o el número de iniciativas que se presentan en cada periodo que nos puede arrojar un número estadístico inmediato, sin embargo, la función principal del congreso local es la actualización del marco normativo estatal, de tal forma, que, aquí podremos observar, analizar y en su momento discutir si los indicadores de la parte cualitativa de la función del legislativo utiliza herramientas aceptables que permitan que esta evaluación le permita al ciudadano realizar juicios de valor derivado de los resultados obtenidos por cada legislatura, lo cual nos pueda ofrecer un punto de comparación entre las mismas de acuerdo a el trabajo que cada una realiza.

En el tránsito y la vigencia que ha tenido la instauración de la evaluación del desempeño del congreso de Sonora, exponemos de manera breve como surge el proyecto de evaluación del trabajo del legislativo, es decir su fundamento legal y quienes fueron los encargados de 
evaluar la función legislativa. De igual forma, se describen los indicadores utilizados en dos épocas distintas en las cuales fungieron dos comités evaluadores distintos, y también mostraremos los resultados obtenidos que nos puedan dar respuesta a la pregunta planteada ¿calidad o cantidad en la presentación de las iniciativas de ley y decreto en el congreso local? De acuerdo al análisis de los resultados que se hayan dado en las legislaturas que fueron evaluadas podremos aventurarnos a emitir un juicio para asegurar si en el congreso sonorense, a partir de la implementación del sistema de evaluación del desempeño legislativo se privilegia la calidad y la profesionalización de la función legislativa.

\section{Marco teórico}

\section{Marco de referencia}

La implementación de la evaluación del desempeño legislativo es un fenómeno y una práctica que ha venido cobrando una relativa importancia en el espectro gubernamental en nuestro país en mayor medida al menos los últimos 15 años. Históricamente, el legislativo rara vez fue objeto de estudio en cuanto a las capacidades de los congresos como entes colegiados y como organizaciones compuestas por individuos que al igual que el resto de las organizaciones desempeñaban un trabajo susceptible de medición.

Por mucho tiempo los congresos locales en México se advertían como entes oscuros donde habitaban los diputados a quienes se les veía como funcionarios políticos que se les daban de premio una curul por favores políticos hechos en campañas, o por haber fungido como líderes de organizaciones gremiales magisteriales u obreras. A partir de lo que se podría considerar la ola democratizadora en América Latina (Huntington, 1991) que deriva en la transición democrática en México (Woldenberg, 2012), los gobiernos locales y las legislaturas en los estados entran en la era de la transparencia. No obstante, a mediados de los noventa aún estaba en embrión el proyecto, y se observaba un legislativo dominado por el ejecutivo y con carencias en aspectos de trasparencia.

Con la entrada de partidos de oposición al PRI a los congresos locales, la función del legislativo, que otrora era algo que el ciudadano común no comprendía bien, pasó a ser una función que se puso en vitrina, antes del término del siglo $\mathrm{XX}$ poco a poco los ciudadanos comenzaron a enterarse de las funciones específicas del legislativo estatal, de tal forma que la entrada de la democracia trajo consigo entre otras cosas, reclamos por parte del ciudadano de transparentar la función del congreso.

A finales de la década de los noventa del siglo anterior, se comenzó a gestar la idea de aplicar indicadores que evaluaran el desempeño de los diputados en el congreso. La evaluación del desempeño siempre fue una herramienta útil en las empresas privadas, ya que se utilizaba como un mecanismo para darle seguimiento y medir el desempeño de los trabajadores en una organización. La medición del desempeño atiende la posibilidad de valorar la productividad individual, la eficiencia, la dedicación, el empeño y demás atributos que se considera deseable en un trabajador (French, 1991). Desde esta perspectiva, la evaluación del desempeño aplicada al congreso, nos presenta un enfoque distinto, ya que la evaluación se realiza a un ente de gobierno que por antonomasia el producto de su función genera resultados de corte principalmente cualitativo, por lo que primero se deben de definir bien cuáles son los indicadores que habrán de utilizarse en la evaluación.

También al pensar en evaluar la actuación del legislativo local nos preguntamos ¿Qué pasa cuando un 
concepto de medición que se aplica en la empresa privada se traslada al sector público? No lo sabemos a ciencia cierta, ni sabemos si el rendimiento y cumplimiento de sus labores permiten al legislador hacer un aporte o ganancia para la organización (Pontifes, 2002) en la búsqueda de cumplir con las metas planeadas, de ahí la intención del estudio del presente trabajo. Por otro lado, a partir de la entrada de las evaluaciones legislativas se ha generado un debate académico y político sobre cuáles son los mejores criterios que permita realizar una adecuada evaluación al legislativo, es decir, se pretende establecer una metodología aceptada que permita aproximarnos a un escenario de evaluación efectiva sobre los resultados de la actuación de los congresos locales.

La discusión y lo que podríamos mencionar como la exigencia de que los organismos legislativos debían ser evaluados lo podemos situar en el año 2002, en ese momento la Confederación Patronal de la República Mexicana (COPARMEX) realizó una encuesta de opinión sobre la actuación del legislativo federal en México, lo que derivó en un documento que fue enviado por dicha organización patronal al congreso llamado 10 propuestas para el fortalecimiento del congreso.

Luis Carlos Ugalde ${ }^{1}$ (CIDE), en el año 2002 realizó un trabajo metodológico que le permitió medir el desempeño legislativo de los diputados del Congreso de la Unión. Ugalde finalmente propone cinco indicadores mínimos para medir el trabajo del congreso: a) dictámenes a favor, b) dictámenes ante el Pleno, c) iniciativas, d) votaciones con alianzas, y, e) iniciativas en temas específicos. A través de este método Ugalde encuentra un modelo sencillo para analizar de manera cuantitativa el trabajo de las y los diputados.

Al parecer 2002 significó un punto de inflexión, ya que el Centro de Investigación para el Desarrollo de México (CIDAC desarrolla el proyecto En contacto con tu diputado, el cual consistía que a través de un portal de Internet cualquier ciudadano interesado podía consultar y conocer el porcentaje de asistencia de su diputado a las sesiones del pleno, así como el sentido de su votación en las leyes aprobadas durante la LVIII Legislatura federal.

Por su parte, Benito Nacif investigador del Centro de Investigación y Docencia Económicas (CIDE), retoma lo hecho por Luis Carlos Ugalde, y presenta un proyecto más completo metodológicamente, patrocinado por el mismo CIDE y por la Fundación Naumman ${ }^{2}$, en lo que podríamos reconocer como un verdadero sistema de evaluación al desempeño legislativo, mejor diseñado y aplicable en este caso para el Congreso de la Unión.

Benito Nacif coordinó un grupo de académicos que se propusieron implementar una herramienta que les permitiera a los ciudadanos informarse del trabajo generado por los diputados y senadores del congreso federal. Derivado de este ejercicio emiten un informe semestral sobre temas de transparencia el cual aborda actividades legislativas haciendo análisis al término de cada periodo de sesiones. Este grupo de académicos se centra en seis aspectos los cuales consideran deben ser monitoreados para conocer si la actividad del congreso va en cumplimiento de su misma función. Los indicadores propuestos son: 1. Función administrativa, 2. Información financiera, 3. Labor legislativa, 4. Controles internos 5. Contrataciones, concesiones y permisos, y, 6. Archivo histórico y servicios de biblioteca.

\footnotetext{
${ }^{1}$ Luis Carlos Ugalde es director general de Integralia Consultores. Fue consejero presidente del IFE (Instituto Federal Electoral) entre 2003 y 2007. Ha sido profesor e investigador en universidades de México y EU y es comentarista en radio y TV. En palabras de Ugalde comenta el Congreso mexicano es una de las instituciones políticas más opacas del país; la opinión pública guía su discusión y su evaluación por experiencias anecdóticas.

${ }^{2}$ La Fundación Friedrich Naumann para la Libertad ofrece propuestas de formación política basadas en la idea del liberalismo, tanto en Alemania como también en todo el mundo, disponible en: https://fnst.org/content/la-fundacion-friedrich-naumann-para-la-libertad.
} 
También, el Instituto Tecnológico de Monterrey (ITESM), a través del Centro de Análisis y Evaluación de la Política Pública (CAEP), derivado de una petición del Congreso de Nuevo León, realizó un ejercicio de análisis del desempeño legislativo en el año 2003. El documento del ITESM realiza una introspección muy completa de los actores que participan, de igual forma retoma algunas variables susceptibles de medición como la escolaridad de los diputados, faltas y asistencias, género, iniciativas presentadas, intervenciones en tribuna, leyes y decretos aprobados, comparativo de sueldos, entre otros. Hasta aquí podemos mencionar los esfuerzos que se habían hecho principalmente en el ámbito federal por tratar de implementar un sistema que permitiera evaluar el trabajo de los congresos.

\section{Evaluación legislativa en Sonora}

En el Estado de Sonora, la organización denominada Sonora Ciudadana, en el año 2006 implementó un sistema de monitoreo legislativo el cual le daba seguimiento a las iniciativas que presentaban los diputados. La metodología que se usó revisaba la tasa de logro de las iniciativas, es decir, realizaba un conteo de las iniciativas presentadas y obtenía el porcentaje de las que lograban ser aprobadas. De igual forma, llevaba un registro de la efectividad de las asistencias de los legisladores a las sesiones del pleno. Este sistema entregaba un informe anual ante la ciudadanía y se mantuvo vigente hasta el año 2010.

De manera paralela, periódicos locales como El Imparcial $^{1}$ publicaban en su portal de internet un sistema en el cual llevaban un conteo de las iniciativas presentadas por las y los diputados. Era un sistema un tanto rústico, ya que no ofrecía gran número de estadísticas o se análisis, se ofrecía como un recurso más bien descriptivo en ocasiones a manera de crónica de los que sucedía en las sesiones del legislativo local. Estos esfuerzos se dieron antes de la llegada en masa de las redes sociales, las cuales posteriormente ofrecían un foro abierto pero en muchos casos subjetivo de quienes publicaban algunos datos que generaba el congreso local.

Todas las evaluaciones y los ejercicios que hasta ese momento se habían hecho, se daban desde la sociedad que no pertenecían y que no estaba financiada por el sector público, y fue el mismo congreso sonorense quien le imprimió la institucionalidad que se requería para que las evaluaciones tomaran un carácter de tipo oficial con datos emanados del propio legislativo que ofrecieran con base en reglas establecidas una evaluación del desempeño con carácter más oficial.

\section{Primera etapa de evaluación}

La LVIII Legislatura 2006-2009, en el año 2007 estaba analizando y discutiendo la propuesta de Ley Orgánica del mismo congreso. Una de las decisiones de esa legislatura fue incorporar en la parte expositiva un título relativo al tema de la evaluación del desempeño legislativo. Como punto principal, el cual sustentaba la propuesta, se expuso que el congreso, a partir de la aprobación de la ley, contaría con un sistema de evaluación de su trabajo legislativo, el cual como misión principal era dar a conocer a la población el desempeño de cada legislatura ${ }^{2}$, agregando que el sistema iba a contribuir en la detección de necesidades y áreas de oportunidad del legislativo, esto con el fin de propiciar su transparencia y mejora continua.

El mecanismo de evaluación seria que, a través de un comité ciudadano, el cual se integraría por al menos 5 personas de la sociedad civil que reunieran requisitos

\footnotetext{
${ }^{1}$ Diario El Imparcial, periódico con mayor tiraje en el estado de Sonora, disponible en: https://www.elimparcial.com/sonora/

2 Artículo 172 de la Ley Orgánica del Poder Legislativo del Estado de Sonora, disponible en: http://www.congresoson.gob.mx/Transparencia/Leyes\#
} 
principalmente de apartidismo político, que fueran profesionistas con sentido crítico serían los encargados de proponer los indicadores de evaluación, y a su vez, ellos serían quienes dieran a conocer los resultados de las evaluaciones las cuales serían anuales ${ }^{1}$. En ese momento, ningún congreso local incluía en sus leyes orgánicas la figura de la evaluación del desempeño legislativo, de tal forma que fue un instrumento y una herramienta muy novedosa en ese momento.

Para elegir al comité ciudadano evaluador, se estableció en el Artículo 174 de la misma Ley Orgánica, que, la Comisión de Régimen Interno y Concertación
Política $^{2}$ (CRICP) tenía la facultad de proponer ante el pleno a por lo menos cinco personas que integrarían el comité ciudadano, el cual duraría en funciones tres años. En el mes de junio de 2008, el pleno del congreso aprobó la designación de nueve ciudadanos propuestos por la CRICP quienes estarían en funciones para el periodo 2008-2011.

La CRICP le encomendó al comité ciudadano que hiciera una propuesta de indicadores de medición, los cuales se utilizarían para evaluar la función del congreso, el comité propuso una batería de siete indicadores con sus respectivos subindicadores, tabla 1 .

Tabla 1. Indicadores de evaluación del desempeño legislativo 2008-2011

\begin{tabular}{|c|c|c|}
\hline No. & Indicador y Subindicadores & Definición \\
\hline \multirow{6}{*}{1} & Iniciativas Enviadas & \\
\hline & Por fracción & No. de Iniciativas por Fracción / No. de Dip. por fracción \\
\hline & Por diputado & Iniciativas por Dip. / No. de Inic. totales de la Legislatura \\
\hline & Del ejecutivo & Inic. ejecutivo / Total de iniciativas \\
\hline & Del poder judicial & Inic. Poder Judicial / Total iniciativas \\
\hline & Ayuntamientos & Inic. por Ayuntamiento / Total de Iniciativas \\
\hline \multirow{4}{*}{2} & Eficiencia legislativa & \\
\hline & Rendimiento legislativo & No. de Inciativas dictaminadas /No. de Iniciativas presentadas \\
\hline & Rezago legislativo & $\begin{array}{l}\text { No. Inic. pend. de dictamen del periodo. / No. Total de Inic. } \\
\text { presentadas del periodo }\end{array}$ \\
\hline & Rendimiento legislativo Contractual & No. Total de Inic. contractuales / Inic. pend. contractuales \\
\hline
\end{tabular}

\footnotetext{
${ }^{1}$ De acuerdo a lo que establece el artículo 41 de la Constitución Política de Sonora, el congreso tiene dos periodos ordinarios de sesiones, del $1^{\circ}$ de septiembre al 15 de diciembre y el segundo de abril a junio. A partir de 2016 el segundo periodo de sesiones comprende del $1^{\circ}$ de febrero al 30 de abril.

${ }^{2}$ La CRICP es la comisión del congreso de Sonora que se integra por los coordinadores parlamentarios representados en el legislativo, es la comisión que analiza los asuntos más importantes y por mayoría de votos deciden a cuáles asuntos darles más celeridad.
} 
Tabla 1. Continuación...

\begin{tabular}{|c|c|c|}
\hline No. & Indicador y Subindicadores & Definición \\
\hline 3 & Asistencia a reuniones del pleno & Entradas al Pleno / Total de sesiones \\
\hline 4 & Asistencia a comisiones & Entradas / Total de reuniones \\
\hline 5 & $\begin{array}{l}\text { Seguimiento de la agenda } \\
\text { legislativa común }\end{array}$ & Asuntos logrados / Asuntos prometidos \\
\hline 6 & Código de conducta & Se Cumple / No se Cumple \\
\hline \multirow{3}{*}{7} & $\begin{array}{l}\text { Índice de ciudadanización de } \\
\text { iniciativas }\end{array}$ & \\
\hline & \multirow[t]{2}{*}{ Elementos/características } & $\begin{array}{l}\text { 1.- Se haya explorado con organismos de la sociedad civil, } \\
\text { organizaciones no gubernamentales, colegios o expertos, } \\
\text { revisión académica }\end{array}$ \\
\hline & & 2.- Que haya tenido un proceso de consulta pública \\
\hline
\end{tabular}

Fuente: Elaboración propia, con base en la gaceta parlamentaria No. 162 del 20 de diciembre de 2008.

De acuerdo a la tabla anterior, los indicadores evaluarían:

1. Iniciativas enviadas. - Este indicador llevaría el registro de las iniciativas enviadas por las fracciones parlamentarias, los mismos legisladores, el ejecutivo, el poder judicial y los ayuntamientos. Se obtenía una tasa de logro dividiendo el número de iniciativas enviadas entre el total de las iniciativas, excepto en las iniciativas enviadas por las fracciones, las cuales se dividían entre el número de legisladores de las fracciones solamente.

2. Eficiencia legislativa. - Este indicador se obtenía dividiendo el total de iniciativas dictaminadas y aprobadas entre el total de iniciativas enviadas. En ese mismo indicador también se obtenía la tasa de rezago legislativo dividiendo las iniciativas pendientes de dictamen entre el total de iniciativas presentadas durante un mismo periodo, se tenía que hacer la precisión ya que existían iniciativas rezagadas de anteriores legislaturas, las cuales se obtenían dividiéndolas solo sobre el total de las iniciativas pendientes de dictamen de otras legislaturas.

3. Asistencia a reuniones del pleno. - Se obtenía dividiendo el número de asistencias de los legisladores entre el número de sesiones del periodo.

4. Asistencia a reuniones de comisiones. - Se obtenía dividiendo el número de asistencia a comisiones entre el número de reuniones de 
comisión, en este caso la asistencia se ponderaba al número de comisiones a las que perteneciera cada diputado $(\mathrm{a})^{1}$.

5. Seguimiento de la agenda común. - Este se obtenía dividiendo los asuntos logrados entre los asuntos presentados previamente en la agenda.

6. Código de conducta. - La ley Orgánica establecía en sus artículos del 24 al 26 el código de conducta, era de corte cualitativo y se esperaba una respuesta dicotómica de 1 en caso de haber violación al código o de cero en caso contrario.

7. Índice de ciudadanización de iniciativas. Este indicador ponderaba si la propuesta legislativa había tenido un proceso de revisión académica o consulta pública antes de su aprobación. En el trabajo previo a la iniciativa se consideraban las reuniones exclusivas que organizan los diputados para atender el análisis $\mathrm{y}$ las aportaciones que pueda hacer los conocedores de temas específicos en relación a alguna iniciativa que hayan presentado o estén por presentar ante el Pleno del Congreso. Para este indicador no se consideran foros actos proselitistas como lo son entrega de fondos económicos o en especie por parte de los legisladores $\mathrm{ni}$ tampoco las reuniones de comisiones ordinarias ya que estas se registran en otro indicador.

El comité ciudadano designado en la LVIII Legislatura se enfrentó a una legislatura intermedia con avance en sus trabajos, de tal forma que logró evaluar dos años de trabajo. Hay que comentar que la aceptación de parte de los diputados de ser sometidos a un proceso de evaluación, en principio no fue bien recibida por todos sus integrantes quienes cuestionaban duramente el método de evaluación o simplemente era desconocimiento de las nuevas herramientas aplicadas a una nueva cultura de transparencia y rendición de cuentas.

El resultado del primer año evaluado que comprendió de septiembre de 2009 a septiembre de 2010 fue:

- Se presentaron 76 iniciativas de ley o decreto, de las cuales 53 las genero el mismo legislativo, 20 de diversos ayuntamientos y 3 del ejecutivo. esto nos arroja un porcentaje del 70\%, 26\% y $4 \%$ respectivamente.

- En cuanto a la eficiencia legislativa se aprobaron 40 iniciativas de las 76 presentadas, lo que nos arroja una tasa del $51 \%$ con un respectivo $49 \%$ de rezago legislativo.

- La asistencia al pleno presento un promedio de $99 \%$, pero se dio en mayor medida porque los legisladores al justificar sus inasistencias no se contabilizaban, situación que cambio en el segundo año de evaluación.

- El indicador de asistencia a las reuniones de comisiones arrojo un promedio de asistencia de $86.38 \%$, con picos de entre 35\% y $0 \%$ de inasistencias.

- La agenda legislativa común no fue posible evaluarla, ya que no se presentó una agenda la cual se pudiera evaluar, los temas los iban presentando de manera espontánea sin haber hecho una planeación legislativa.

- No se presentaron violaciones al código de conducta.

\footnotetext{
${ }^{1}$ Había diputados que formaban parte de 16 comisiones y quienes pertenecían a 4, de tal forma que entre mayor era el número de comisiones a las que se perteneciera cada inasistencia tenía un porcentaje menor de afectación.
} 
- El índice de ciudadanización de las iniciativas no arrojo un dato cuantitativo ya que el indicador carecía de una fórmula la cual nos arrojara un resultado contundente.

Utilizando los mismos criterios de evaluación, en el segundo año de ejercicio evaluativo, que comprende de septiembre de 2010 a septiembre de 2011, fueron presentadas 71 iniciativas de ley o decreto, de las cuales 69 las generó el mismo legislativo y 2 el ejecutivo. Esto nos arroja un porcentaje del $97 \%$ y $3 \%$ respectivamente. En cuanto a la eficiencia legislativa se aprobaron 46 iniciativas de las 71 presentadas, lo que nos arroja una tasa del $65 \%$ con un respectivo $35 \%$ de rezago legislativo.

La asistencia al pleno presentó un promedio de 98\%, en esta segunda evaluación se agregó la información de las faltas justificadas ya que en la anterior evaluación la posibilidad de justificar las inasistencias distorsionaba los porcentajes de ese indicador, la evaluación incluyó una tabla en la cual se promedió el número de inasistencias en relación al número de diputados de cada fracción parlamentaria, siendo el partido Nueva Alianza el que promedió el número más alto con 6.33 faltas, y el PRD el menor con un promedio de 2 faltas por diputado. Sin embargo, en relación al número de diputados, la mayor efectividad fue del partido del PAN, ya que logro un promedio de 2.21 inasistencias en promedio con 14 diputados en el mismo número de sesiones.

El indicador de asistencia a las reuniones de comisiones arrojó un promedio de asistencia de 86.38\%, con picos de entre 35\% y $0 \%$ de inasistencias. En el caso de las inasistencias a las reuniones de comisión, se permitía que el legislador presentara algún justificante que excusara la falta, los cuales generalmente eran de carácter médico, o por atender algún llamado a alguna otra reunión en otro lugar fuera del recinto legislativo o fuera de la ciudad.
La agenda legislativa común no fue posible evaluarla, ya que no se presentó una agenda la cual se pudiera evaluar, los temas los iban presentando de manera espontánea sin haber hecho una planeación legislativa. En el caso del código de conducta no se registraron violaciones al mismo.

El índice de ciudadanización de las iniciativas o de trabajo previo a la iniciativa, no registró un dato cuantitativo, ya que el indicador carecía de una fórmula la cual nos arrojara un resultado contundente. En ese sentido se registraron reuniones de las distintas comisiones, en las cuales esporádicamente se invitaba a algún funcionario público que conociera del tema o se registraba la realización de algún foro de consulta, estos no eran muy frecuentes ya que la cantidad de recurso imposibilitaba que la mayoría de los diputados tuvieran la opción de realizar dichos foros. Dentro de las actividades previas a la aprobación de diversas iniciativas, se destaca que el Congreso registró en el área de eventos especiales la organización de 10 foros, de los cuales 9 fueron promovidos por Damián Zepeda Vidales (PAN) y uno por Vicente Solís Granados (PRI) como los únicos diputados que promovieron foros de consulta ciudadana.

La primera etapa de evaluación fue con una metodología de carácter político más que de orden cuantitativo, en el caso de las iniciativas al medir si se aprobaba o no, se dejaba a consideración de los votos de las comisiones si esta pasaba el tamiz de análisis para presentarse en el pleno, esto sin importar si la iniciativa cumplía con requisitos de profesionalismo o no, todo se dejaba a la construcción de mayorías. De igual forma los números de las asistencias o inasistencias servía para noticias o encabezados periodísticos para exhibir a los diputados faltistas. Por otra parte, no existía una fórmula que nos ofreciera un número de evaluación en alguna escala de logro, el cual pudiera comparar el rendimiento 
de un periodo frente a otro. De igual forma, el indicador de trabajo previo a la iniciativa, no ofrecía una valoración cuantitativa de cualquier trabajo que se haya hecho en la configuración de la misma, y solo se limitaba a la descripción anecdótica de quien había invitado a alguna persona a los trabajos de comisiones o a registro de algún foro de consulta.

\section{Segunda etapa de evaluación}

El primer comité ciudadano evaluador detuvo sus funciones en 2011, lo que le permitió evaluar solo dos periodos anuales de la LIX Legislatura (2009-2012), de tal forma que la LIX Legislatura terminó su último año de funciones sin una evaluación final. A pesar de que el sistema de evaluación del desempeño legislativo estaba establecido en la ley orgánica del mismo congreso, este último decidió no convoca la integración de un nuevo comité ciudadano. De igual manera, la LX Legislatura (2012-2015) decidió no convocar en todo el tiempo de sus funciones la conformación de un nuevo comité ciudadano, esto a pesar de que la ley obligaba a que el congreso contara con este novedoso sistema de evaluación, las y los diputados simplemente decidieron que no querían ser evaluados.

La LXI Legislatura (2015-2018), al entrar en funciones, decidió que se debían de retomar los esfuerzos de la evaluación legislativa y en diciembre de ese mismo año aprobó un acuerdo en el pleno para lanzar una convocatoria a los interesados en evaluar la función del poder legislativo en Sonora.
El 17 de marzo de 2016, el pleno del congreso aprobó la designación de cinco ciudadanos que habrían de fungir como evaluadores del legislativo por los siguientes tres años ${ }^{1}$. Los ciudadanos que fueron designados propuestos por la CRICP y aprobados por el pleno representaban a distintas áreas de la sociedad sonorense ya que emanan del sector empresarial, industrial, académico, ciudadano y magisterial. Una vez elegidos tomaron protesta de ley el día 29 de marzo de 2016 ante el mismo Congreso.

El nuevo comité evaluador decidió que no operaría con los indicadores utilizados por el antiguo comité, ya que dichos parámetros obedecían a un contexto distinto al que se vivía en ese momento. Una de las primeras acciones del nuevo comité ciudadano fue cambiar los criterios de evaluación, y en para esa ocasión el comité pidió la participación de otros actores de la sociedad para poder definir de manera plural y de acuerdo a la inquietud de los ciudadanos cuáles serían los indicadores que plasmaran mejor el sentir de la población.

Para tal propósito, el comité ciudadano definió una agenda de consulta (tabla 2) en la cual se convocó al sector empresarial, a un grupo académico y a los mismos diputados para preguntarles la forma en la cual debería ser evaluado el trabajo legislativo sonorense. Derivado de lo anterior se programaron varias reuniones con los actores mencionados.

${ }^{1}$ Gaceta marzo 16, 2016. Año 9, No. 795, disponible en: http://www.congresoson.gob.mx:81/api/ServiceDos?id=2531. 
Tabla 2. Agenda de consulta para definir indicadores de evaluación del desempeño legislativo

\begin{tabular}{|c|c|l|}
\hline No. & Fecha & \multicolumn{1}{|c|}{ Grupo } \\
\hline 1 & $27-a b r-16$ & Fracción PAN \\
\hline 2 & $03-$ may-16 & Fracción PRI \\
\hline 3 & 11 -may-16 & $\begin{array}{l}\text { Fracción Nueva Alianza, PRD, MC, } \\
\text { MORENA E independientes }\end{array}$ \\
\hline 4 & 18 -may-16 & Empresarios \\
\hline 5 & $25-$ may-16 & Académicos \\
\hline
\end{tabular}

Fuente: Elaboración propia, con información de la gaceta legislativa 849, 2016.

De acuerdo al comité, el propósito de las reuniones era que los indicadores y los criterios de evaluación fueran congruentes con las necesidades de información que reclamaban varios representantes de la sociedad. Los diputados, los empresarios y los académicos en las distintas reuniones manifestaron cuales debían ser los indicadores ideales para evaluar al legislativo, tabla 3. Fueron muchas las ideas expresadas por los distintos grupos, los diputados, por ejemplo, mencionaron evaluar la calidad de las iniciativas, establecer mayores controles en la entrega-recepción de las legislaturas, evaluar la participación ciudadana en las iniciativas, en términos generales evaluar mejor todo el proceso legislativo desde el principio hasta el final.

Los empresarios manifestaron que el congreso debía configurar leyes útiles a la sociedad, es decir evitar lo que se conoce como letra muerta o leyes que no responden a ninguna necesidad de la población, un mejor flujo de información entre el congreso y los ciudadanos, claridad y simplicidad de las leyes evitando demasiada retórica, evaluar mejor la transparencia, incluir la evaluación de la 3 de $3^{1}$, así como evaluar si cumplen las metas establecidas en la agenda legislativa.

De parte del sector académico (tabla 3), entre las propuestas más interesantes se planteó evaluar la calidad, eficiencia e impacto de las leyes, evaluar la transparencia del mismo congreso, evaluar los sistemas de información del legislativo como publicar los temas del congreso con antelación, evaluar la relación del ejecutivo frente al legislativo, así como evaluar la fiscalización.

\footnotetext{
${ }^{1}$ De acuerdo a lo que se publica en la página https://www.3de3.mx/, se establece la obligación de todos los funcionarios públicos de hacer públicas tres declaraciones: declaración patrimonial, de intereses y fiscal. Además, define reglas claras de conducta para los servidores públicos y actores privados, así como sanciones para los corruptos.
} 
Tabla 3. Resumen de propuestas de diversos sectores para evaluar al legislativo en Sonora

\begin{tabular}{|c|c|c|}
\hline No. & Sector & Variables \\
\hline \multirow{18}{*}{1} & \multirow{18}{*}{ Diputados } & Entrega-recepción de comisiones \\
\hline & & Evaluar el presupuesto \\
\hline & & Mejorar calidad de iniciativas \\
\hline & & $\begin{array}{l}\text { Evaluación diferenciada diputados } \\
\text { electos/plurinominales }\end{array}$ \\
\hline & & Darle mayor/menor importancia a los exhortos \\
\hline & & Evaluar el proceso legislativo tiempos/eficiencia \\
\hline & & Evaluar la agenda legislativa \\
\hline & & Evaluar participación ciudadana \\
\hline & & Evaluar el impacto de las leyes \\
\hline & & Evaluar gestión en los distritos \\
\hline & & Evaluar la tasa de éxito de las iniciativas \\
\hline & & $\begin{array}{l}\text { Evaluar aspectos cualitativos de los trabajos de las } \\
\text { comisiones }\end{array}$ \\
\hline & & Evaluar lo que establece la ley orgánica \\
\hline & & Evaluar los compromisos de campaña \\
\hline & & Evaluar la participación en el Pleno y comisiones \\
\hline & & Evaluar el lenguaje de las iniciativas \\
\hline & & Evaluar la eficiencia de las comisiones \\
\hline & & Evaluar los informes del diputado \\
\hline
\end{tabular}


Tabla 3. Continuación...

\begin{tabular}{|c|c|c|}
\hline No. & Sector & Variables \\
\hline \multirow{11}{*}{2} & \multirow{11}{*}{ Empresarios } & Crear leyes útiles \\
\hline & & Mejor flujo de información entre ciudadano y diputado \\
\hline & & Evaluar consultas públicas \\
\hline & & Claridad y simplicidad de las leyes \\
\hline & & Evaluar la transparencia, presupuesto, 3 de 3. \\
\hline & & Evaluar las comisiones \\
\hline & & Evaluar la ética y el comportamiento del diputado \\
\hline & & Evaluar si se reúne con sus representados \\
\hline & & Evaluar si se logran las metas \\
\hline & & $\begin{array}{l}\text { Evaluar si se cumplen las disposiciones del legislativo } \\
\text { por el ejecutivo }\end{array}$ \\
\hline & & Evaluar pertinencia de los exhortos \\
\hline \multirow{5}{*}{3} & \multirow{5}{*}{ Académicos } & Evaluar la relación del legislativo/ejecutivo \\
\hline & & $\begin{array}{l}\text { Evaluar calidad, eficiencia e impacto de las } \\
\text { leyes/iniciativas }\end{array}$ \\
\hline & & Evaluar metas \\
\hline & & Evaluar la transparencia del mismo Congreso \\
\hline & & Evaluar rendición de cuentas \\
\hline
\end{tabular}


Tabla 3. Continuación...

\begin{tabular}{|l|l|l|}
\hline No. & Sector & Variables \\
\hline \multirow{3}{*}{3} & \multirow{5}{*}{ Académicos } & Medir la independencia del legislativo \\
& & $\begin{array}{l}\text { Medir la capacidad institucional del legislativo para } \\
\text { cumplir su función }\end{array}$ \\
\cline { 3 - 4 } & Seguimiento a las leyes \\
\cline { 3 - 4 } & Evaluar la fiscalización \\
\cline { 3 - 4 } & Evaluar que la evaluación corrija algún problema \\
\hline
\end{tabular}

Fuente: Elaboración propia, con información de la gaceta legislativa 849, 2016.

El comité ciudadano, después de recoger los puntos de vista de los distintos actores convocados para definir los indicadores de medición propuso tres grandes indicadores que a su vez de desagregaban en varios subindicadores.
Se propuso un indicador de calidad del proceso legislativo, uno de desempeño básico y un último de la calidad de la información que atendía a los asuntos de transparencia tal como se apreciar en la tabla 4.

Tabla 4. Indicadores de evaluación legislativa aprobados en el año 2016

\begin{tabular}{|c|l|l|c|}
\hline \multicolumn{1}{|c|}{ Indicador } & \multicolumn{1}{|c|}{ Subindicador } & $\begin{array}{l}\text { Ponderación Máxima del } \\
\text { Subindicador }\end{array}$ & $\begin{array}{c}\text { Ponderación Máxima } \\
\text { del Indicador }\end{array}$ \\
\hline Calidad del proceso & Proceso. & $20 \mathrm{Pts}$ & \multirow{2}{*}{100 Pts. } \\
\hline Legislativo & Fundamentación. & $40 \mathrm{Pts}$ & \\
\hline & Vinculación. & $40 \mathrm{Pts}$ & \\
\hline
\end{tabular}


Tabla 4. Continuación...

\begin{tabular}{|c|c|c|c|}
\hline Indicador & Subindicador & $\begin{array}{l}\text { Ponderación Máxima del } \\
\text { Subindicador }\end{array}$ & $\begin{array}{c}\text { Ponderación Máxima } \\
\text { del Indicador }\end{array}$ \\
\hline \multirow{6}{*}{ Desempeño básico } & Asistencia a comisiones. & $\%$ & \multirow{6}{*}{$100 \%{ }^{*}$} \\
\hline & Asistencia al Pleno. & $\%$ & \\
\hline & Faltas justificadas. & Descriptivo & \\
\hline & $\begin{array}{l}\text { Cumple el Código de } \\
\text { Conducta. }\end{array}$ & Descriptivo & \\
\hline & $\begin{array}{l}\text { Registro de leyes, decretos y } \\
\text { acuerdos. }\end{array}$ & Descriptivo & \\
\hline & Total de iniciativas. & Descriptivo & \\
\hline \multirow{6}{*}{ Calidad de la información } & Currículo actualizado. & 15 Pts & \multirow{6}{*}{100 Pts. } \\
\hline & Declaración 3 de 3. & 20 Pts & \\
\hline & ¿Tiene oficina de enlace? & 15 Pts & \\
\hline & $\begin{array}{l}\text { Tiene información de su } \\
\text { comisión actualizada. }\end{array}$ & 10 Pts & \\
\hline & $\begin{array}{l}\text { Hace boletines informativos y } \\
\text { acciones legislativas. }\end{array}$ & 30 Pts & \\
\hline & $\begin{array}{l}\text { ¿Se conocen las comisiones } \\
\text { de destino? }\end{array}$ & 10 Pts & \\
\hline
\end{tabular}

Fuente: Elaboración propia. 
Con los nuevos indicadores se buscaba en la medida de lo posible, que la función de los legisladores fuera susceptible de una medición cuantitativa y que al final se pudiera emitir una evaluación general del congreso. Uno de los indicadores más interesantes fue el que evaluaba la calidad del proceso legislativo, este indicador evaluaba que las iniciativas enviadas reunieran al menos tres aspectos básicos, que pasaran por un proceso de presentación y formalidad en su presentación ante el congreso, que su redacción y fundamentación tuviera fuentes válidas comprobables, y que la iniciativa pasara por una especie de vinculación o socialización ciudadana, cada uno de los subindicadores era revisado y analizado para otorgarle una evaluación.

La ponderación ofrecía en cuanto al proceso interno un máximo de 20 puntos, la fundamentación hasta 40 puntos y la vinculación ciudadana hasta 40 puntos, lo cual creaba una escala de 100 puntos.

\section{Evaluación 2015-2018}

El comité ciudadano 2016-2019 evaluó los tres periodos anuales correspondientes de la LXI Legislatura. En ese lapso de tiempo revisaron y analizaron 759 documentos entre leyes, decretos y acuerdos. No obstante, los asuntos que se evaluaban en su contenido de acuerdo al indicador de calidad del proceso legislativo solo eran las iniciativas de ley y de decreto, en el caso de los acuerdos estos se revisaban solo para incluir la estadística de los tipos de acuerdos que genero el congreso como parte de los procesos legislativos (tabla 5), podemos observar que en el periodo de 2016-2017 el comité revisó y analizo mayor número de iniciativas.

Tabla 5. Numero de asuntos revisados de la LXI Legislatura

\begin{tabular}{|r|c|c|c|c|c|}
\hline Período & $\begin{array}{c}\text { Iniciativa } \\
\text { de Ley }\end{array}$ & $\begin{array}{c}\text { Decretos } \\
\text { de reforma }\end{array}$ & Acuerdos & $\begin{array}{c}\text { Leyes } \\
\text { federales } \\
\text { armonizadas }\end{array}$ & Totales \\
\hline $2015-2016$ & 27 & 69 & 167 & 2 & 265 \\
\hline $2016-2017$ & 27 & 89 & 174 & 8 & 298 \\
\hline $2017-2018$ & 18 & 81 & 92 & 5 & 196 \\
\hline Total & 72 & 239 & 433 & 15 & 759 \\
\hline
\end{tabular}

Fuente: Elaboración propia.

De igual forma, se advierte que de los temas a evaluados se concentran mayormente en los decretos, que tiene cierta lógica ya que son procesos legislativos que en esencia reforman leyes, a diferencia del caso de las leyes que atienden a reformas de la constitución local, o a cuerpos normativos completos.

Por otra parte, el comité hizo una clasificación de los temas en los cuales el legislativo ponía más atención o en los cuales enviaba mayor número de iniciativas, tabla 6. 
Tabla 6. Distribución temática de las iniciativas evaluadas 2016-2019

\begin{tabular}{|c|c|c|c|c|c|c|}
\hline No. & Temas tratados & 2015-2016 & 2016-2017 & 2017-2018 & Total & $\%$ \\
\hline 1 & $\begin{array}{l}\text { Gobernabilidad, estabilidad y } \\
\text { paz social, justicia, seguridad, } \\
\text { política, anticorrupción, } \\
\text { transparencia y derechos } \\
\text { humanos. }\end{array}$ & 47 & 48 & 47 & 142 & 0.45 \\
\hline 2 & Economía y finanzas públicas. & 8 & 13 & 6 & 27 & 0.09 \\
\hline 3 & $\begin{array}{l}\text { Infraestructura } \\
\text { sustentabilidad. }\end{array}$ & 2 & 12 & 10 & 24 & 0.08 \\
\hline 4 & Políticas para el desarrollo. & 18 & 23 & 23 & 64 & 0.20 \\
\hline 5 & $\begin{array}{l}\text { Grupo transversal para género, } \\
\text { igualdad, grupos vulnerables, } \\
\text { etnias. }\end{array}$ & 11 & 18 & 13 & 42 & 0.13 \\
\hline 6 & $\begin{array}{l}\text { Régimen interno del } \\
\text { Congreso. }\end{array}$ & 12 & 2 & 4 & 18 & 0.06 \\
\hline
\end{tabular}

Fuente: Elaboración propia.

El comité realizó cinco tipos distintos de clasificación de los temas abordados por el congreso, en los cuales destacan los temas asociados a la gobernabilidad de la entidad por encima de temas como economía, infraestructura, grupos vulnerables, educación, salud entre otros. La atención del congreso se centró en un $45 \%$ en temas de gobernabilidad, justicia y derechos humanos.

En cuanto a la calidad del proceso legislativo, que en esencia se podría considerar el indicador base de las evaluaciones del legislativo, ya que la función principal los legisladores es crear o actualizar el marco normativo estatal mostró algunas variaciones. De acuerdo a los datos obtenidos, durante el periodo 2015-2016 del primer año de funciones del congreso sonorense, la calidad del proceso legislativo obtuvo una evaluación de 68.65 de una escala de 100.

Una de las debilidades que se observó en ese momento fue la poca vinculación que tenían los legisladores con los ciudadanos en la presentación de las iniciativas, ya que un máximo de 40 puntos solo 17.31, es decir, que redactaban sus iniciativas y las presentaban, con poca o nula vinculación social. Cabe aclarar, que, a diferencia del comité anterior, en los nuevos criterios no importaba si la iniciativa era aprobada o no, en este caso se le dio más importancia al grado de profesionalismo que le imprimiera el legislador a su proyecto, e hicieron a un lado la eficiencia política. 
Gráfico 1. Calidad del proceso legislativo LXI Legislatura

\section{Comparativo Calidad del proceso Legislativo} 2015-2018

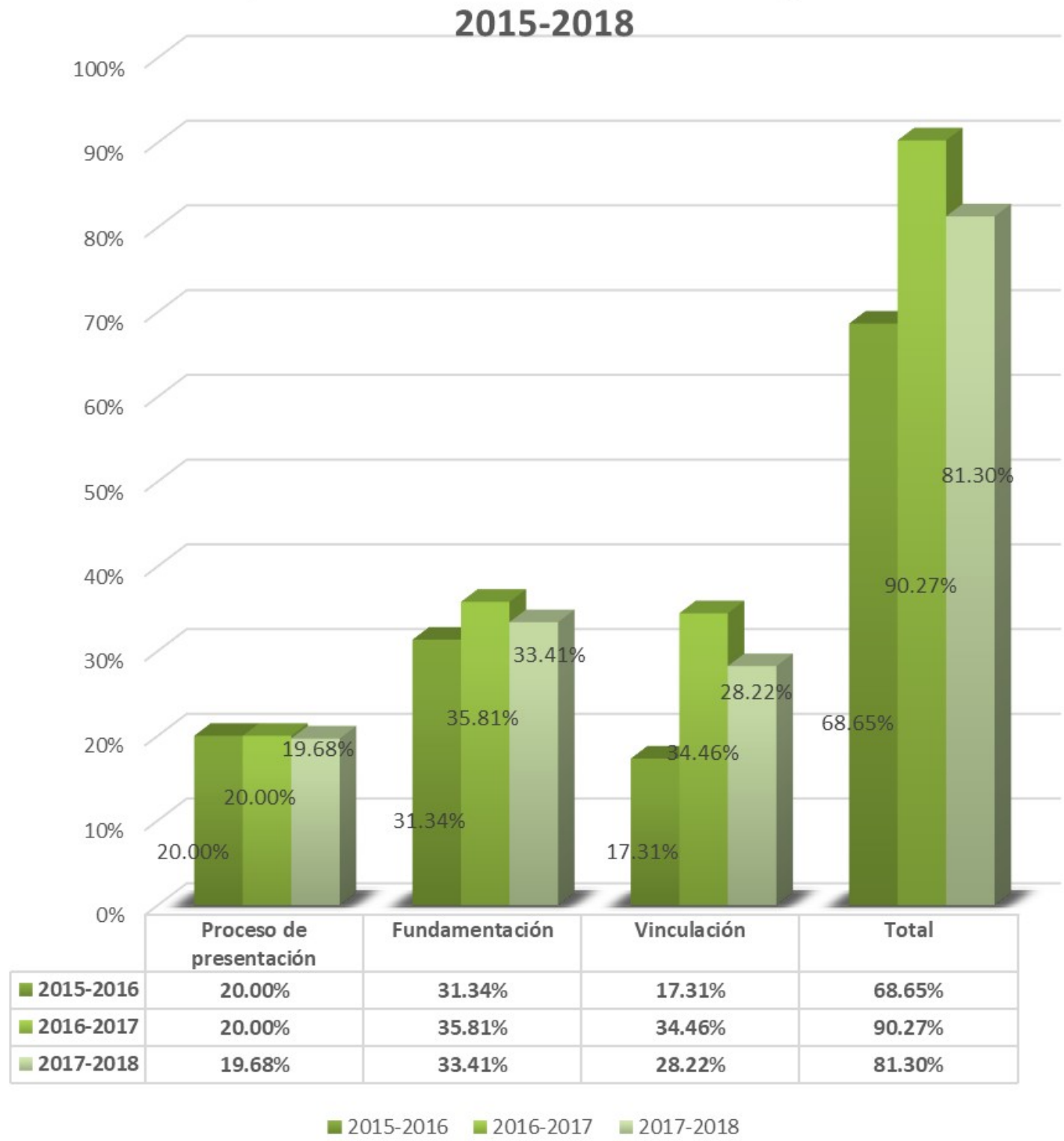

Fuente: Tomado del tercer informe de evaluación del desempeño legislativo de la LXI Legislatura del estado de Sonora. Disponible en: http://www.congresoson.gob.mx/Transparencia/informeComite 
Para el segundo año de trabajo, se observó una mejoría significativa en el rubro de la vinculación social al pasar del primer periodo al segundo de 17.31 a 34.46 , lo que hizo que la evaluación mejorara en términos generales en la calidad del proceso legislativo a 90.27. Esto nos puede indicar que los legisladores, ante esta situación de estar observados por el comité decidieran utilizar los resultados anteriores para imprimir mayor esfuerzo en las áreas que le eran desfavorables. En el último periodo de evaluación, podemos observar una caída de casi 10 puntos porcentuales, de igual forma se puede apreciar que de nueva cuenta el talón de Aquiles de los legisladores es la vinculación social, ya que, de estar por arriba de los 34 puntos, bajo hasta los 28 puntos. Algunos conocedores del tema mencionan que la baja se debió a que al comenzar las campañas electorales en su último tienden a descuidar algunos aspectos de los procesos legislativos.

En el indicador de desempeño básico, el cual contiene el registro de las asistencias al pleno, a comisiones, cumplimiento del código de conducta y el registro de iniciativas presentadas, alcanzaron un promedio de 86.76 en toda la legislatura, teniendo su mayor debilidad en la asistencia a comisiones promediando 81.21 de asistencia. En el indicador de calidad de la información, el cual se enfoca a conocer si los legisladores ofrecen información a la ciudadanía de sus actos y podemos encontrar variables como si el legislador tiene su currículo actualizado, si presento su declaración 3 de 3 , si tiene oficina de enlace, si tiene actualizada la información de las comisiones a las que pertenece, y si presenta informes legislativos anuales, la LXI legislatura alcanzó una evaluación de 80.3, siendo el subindicador de la presentación de informes legislativos anuales la variable que le significo mayor baja en la evaluación.

En lo que respecta a la eficiencia legislativa, el cual se obtiene dividiendo el número de dictámenes de las comisiones entre el número de asuntos que les fueron turnados, la evaluación fue del 57.63, en esta ocasión se hizo un registro y porcentajes de cada comisión, para este estudio tomamos en cuenta las comisiones que recibieron al menos 10 asuntos, de ellas la que mejor porcentaje obtuvo fue la seguridad publica al alcanzar un $60 \%$ de efectividad en la generación de los dictámenes de los asuntos recibidos. Con este indicador se evita que los legisladores hagan uso de la congeladora legislativa, situación en la cual las comisiones se olvidan de revisar los asuntos y se quedan por tiempo indefinido.

\section{Conclusiones}

La evaluación del desempeño legislativo en Sonora ha pasado por dos épocas, la primera en la cual se inserta esta herramienta en un momento en el que los evaluadores no tenían consigo un modelo acabado de cómo era la mejor forma de evaluar una función de corte tan cualitativo como lo es la de legislar. Sin embargo, este primer esfuerzo sirvió para que la curva de aprendizaje poco a poco trajera consigo la misma profesionalización de ciudadanos evaluadores preocupados por el quehacer legislativo de la entidad. De igual forma, en esa primera etapa se advirtió reticencia de parte del congreso a ser sometido a evaluaciones periódicas, ello sucedía, por lógica, al arrastrar vicios de actuaciones de legislaturas anteriores, en las cuales, los asuntos poco se analizaban, y en general el mayor legislador era el ejecutivo estatal el cual generalmente tenía las mayorías necesarias para que le fueran aprobados todos sus proyectos. De igual forma, el mismo gasto del legislativo era una incógnita, ya que era común que gran parte del presupuesto se iba en turismo legislativo en el pago de boletos de avión o en la contratación de servicios sin que nadie vigilara si se hacían las licitaciones de acuerdo a un proceso trasparente, de tal forma, que, al estar vigilados en algunas áreas sintieron que les estaban quitando algunos derechos que de manera histórica les correspondían. 
La segunda etapa de evaluación, trajo consigo la propuesta de indicadores en los cuales se ponía en vitrina la actuación de los diputados con la implementación de indicadores que en gran medida surgieron de la misma sociedad, de tal forma que pudimos advertir en algunos momentos síntomas de mejoramiento en el proceso legislativo ya que los mismos diputados no querían hacerse de mala publicidad en los medios de comunicación como legisladores ineficientes, no obstante los anterior, sigue prevaleciendo en el entorno que sucesos políticos como las elecciones pueden motivar que los legisladores dejen de lado buscar buenas evaluaciones aunque esto se convierta en una debilidad ante el electorado, ya que de cierta manera, ante un ciudadano más sofisticado a la hora de votar puede tener en cuenta las evaluaciones del desempeño legislativo, como un incentivo para votar por la profesionalización de los diputados.

\section{Referencias:}

Dessler, G. y Varela, R. (2011) Administración de recursos humanos. Enfoque latinoamericano. México: Pearson Education. Recuperado de

https://cucjonline.com/biblioteca/files/original/0ee4993 0c54202fa9d631ebce4af2438.pdf.

French, W. L. (1991) La administración de personal, desarrollo de recursos humanos. México: Ed. Noriega Limusa. 2da edición, 3a reimpresión.

Huntintong, S. (1991) La Tercera Ola. Buenos Aires, Argentina: Editorial Paidós.

Mény, I. y Thoening, J. (1992) Las políticas públicas. Barcelona, España: Editorial Ariel.

Ordaz, A. y Rodríguez L. (2012) Evaluación del Desempeño Legislativo. La experiencia del Congreso de Sonora. En Textos Académicos, (Coordinador) Arturo Ordaz Álvarez. México: Universidad de Sonora.
Pontifes, A. (2002): La Evaluación del Desempeño y sus distintos enfoques en la gestión de los Recursos Humanos en las organizaciones. Foro Nacional de Profesionalización y Servicio Público de Carrera. Santiago de Querétaro. México.

Woldenberg, J. (2013) Historia mínima de la transición democrática en México. Revista Mexicana de Derecho Electoral. México: El Colegio de México; Instituto de Investigaciones Jurídicas, Universidad Nacional Autónoma de México-Instituto de Investigaciones Jurídicas. Núm. 3, enero-junio, pp. 519-524.

\section{Leyes:}

Congreso del Estado libre y soberano de Sonora. (2007). Ley 77 Orgánica del Poder Legislativo del Estado de Sonora. México.

Congreso Constituyente de Sonora. (1917 marzo). Constitución Política del Estado Libre y Soberano de Sonora. México.

\section{Páginas de internet:}

Transparencia mexicana. (2016). Iniciativa \#3 de 3. Recuperado de https://www.3de3.mx/

Congreso del Estado de Sonora. (2016). Portal del Poder Legislativo del Estado de Sonora. Recuperado de http://www.congresoson.gob.mx/

Integralia. (2018). Portal Integralia Consultores. Recuperado de

https://integralia.com.mx/web/index.php/publicaciones / 